\title{
STRUCTURE-BASED DETERMINATION OF IMAGING LENGTH FOR SUPER-RESOLUTION LOCALIZATION MICROSCOPY
}

\author{
Kuan-Chieh Jackie Chen ${ }^{1,2}$, Jelena Kovačević ${ }^{1,2,3}$, and Ge Yang ${ }^{1,2}$ \\ ${ }^{1}$ Dept. of Biomedical Eng., ${ }^{2}$ Center for Bioimage Informatics, ${ }^{3}$ Dept. of Electrical \& Computer Eng. \\ Carnegie Mellon University, Pittsburgh, PA 15213, USA
}

\begin{abstract}
Localization-based super-resolution techniques are revolutionizing biological research by breaking the diffraction limit of fluorescence microscopy. Each super-resolution image is reconstructed from a time series of images of randomly activated fluorophores. Here, a fundamental question is to determine the minimal imaging length so that the reconstructed image faithfully reflects the biological structures under observation. So far, proposed methods focus entirely on image resolution, which reflects localization uncertainty and fluorophore density, without taking into account the fact that images of biological structures are structured rather than random patterns. Here, we propose a different approach to determine imaging length based on direct quantification of image structural information using Gabor filters. Experimental results show that this approach is superior over approaches that only account for image-intensity distribution, confirming the importance of using structural information. In contrast to resolution-based methods, our method does not require an artificial selection of image resolution and provides a statistically rigorous strategy for determining imaging length based on image structural information.
\end{abstract}

Index Terms - Super-resolution microscopy, STORM, fluorescence imaging, determining imaging length

\section{INTRODUCTION}

The resolution of conventional optical microscopy is limited by the diffraction of visible light to $\sim 200 \mathrm{~nm}$, the Rayleigh limit. Recent advances in super-resolution microscopy techniques have made it possible to break this limit to reach $\sim 20 \mathrm{~nm}$ in resolution and are revolutionizing biological research by resolving subcellular structures at nanometer resolution.

Localization-based methods such as stochastic optical reconstruction microscopy (STORM) [1] and photoactivated localization microscopy (PALM) [2] achieve super resolution

We thank Dr. Michael Davidson for providing the sample slide used to generate the data shown in Fig. 1. We also gratefully acknowledge support from the US NSF through awards 1017278 (J.K.), and DBI-1149494, DBI1052925, MCB-1052660 (G.Y).
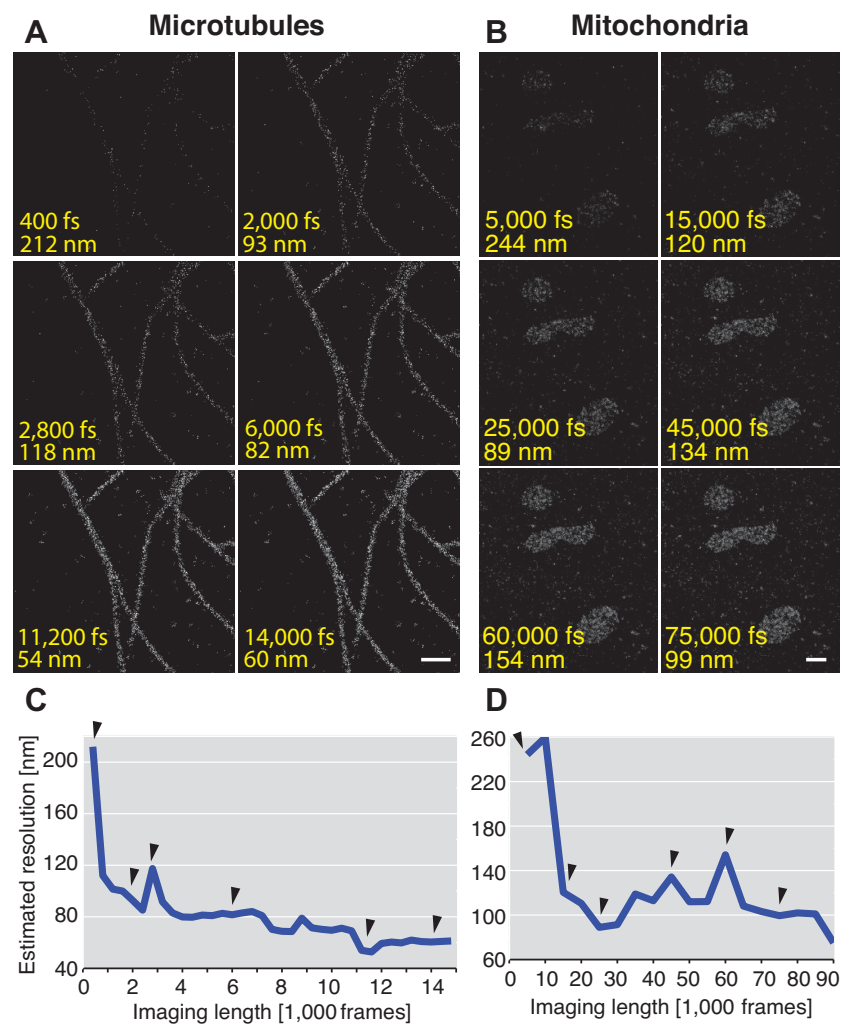

D

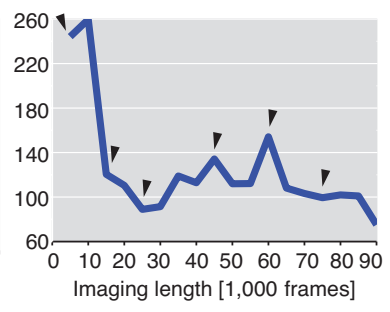

Fig. 1. STORM images of microtubules and mitochondria. (A, B) Reconstructed images with different imaging lengths (the number of frames, fs). (C, D) Estimated resolutions determined by FRC. Black arrows indicate images shown in $(\mathbf{A}, \mathbf{B})$; estimated resolutions are also shown below the frame numbers. Scale bars, $500 \mathrm{~nm}$.

by randomly activating separate fluorophores and computationally determining their locations at nanometer resolution. Each super-resolution image is assembled from a time series of images of randomly activated fluorophores (Fig. 1A, B).

In this paper, we attempt to answer the following fundamental question: What is the minimal imaging length needed for image analysis so that the reconstructed superresolution image faithfully reflects the biological structures under observation? Avoiding unnecessary imaging is essential for many reasons, especially minimizing photodamage. Fig. 1A shows a sequences of STORM images of 
microtubules with different imaging lengths. At 2,000 frames or fewer, the detections are insufficient to provide useful information, while after 6,000 frames the increasing structural information provided from the image is almost identical. Similar observations can be made on images of mitochondria in Fig. 1B. These two examples are representative of a wide variety of $1 \mathrm{D}$ and $2 \mathrm{D}$ biological structures, respectively.

Currently, the common practice is to choose the number of frames to image a priori. This risks acquiring either too few (not enough information) or too many (wasting time or even damaging the imaging samples by photobleaching or photo-toxicity) frames than needed for a given application. Moreover, reducing the imaging length required for a single high-resolution frame is crucial to achieving live imaging.

So far, solutions proposed for this problem focused entirely on image resolution, which reflects localization uncertainty and fluorophore density. A representative of these methods is Fourier ring correlation (FRC), which is used to determine the image resolution from the localized fluorophore data [3, 4]. FRC measures the level of details of reconstructed images. It does not, however, directly consider the structure being imaged.

Specifically, in determining imaging length using FRC, the selection of achieved resolution is arbitrary. For example, although at 2,000 frames, the resolution of 93nm (Fig. 1A) is beyond the Rayleigh limit, the structure of the microtubules is not properly captured. Fig. 1C, D show the resolutions estimated by FRC from the reconstructed images of both examples with different imaging lengths. In particular, the expected microtubule thickness of $25 \mathrm{~nm}$ in the first example (Fig. 1A) is $\sim 60 \mathrm{~nm}$ considering the size of labeling antibodies. From Fig. 1C, FRC suggest to collect 11,000 frames, which is unnecessary since the structure of microtubules stabilizes after 6,000 frames, and similarly for Fig. 1B, D.

In this paper, we present a structure-based method that uses structural information present in the image for determining imaging length. We extract this structural information using the orientation histogram with Gabor filters and perform a statistical test to determine whether information has saturated. Equipped with this method, we develop an acquisition system that determines the imaging length adaptively.

\section{DETERMINING IMAGING LENGTH}

Problem definition For localization-based super-resolution imaging, randomly activated fluorophores from each fluorescent image are first collected. At a given imaging time $t$, a high-resolution image $I_{t}$ is reconstructed using the detections in all frames up to time $t$. The goal of determining imaging length is to extract useful image information and then determine whether there is significant information change between $I_{t}$ and the reconstructed image at the previous time point $I_{t-1}$; if not, imaging stops.
Intensity-based analysis One way of summarizing image information is to compute image-intensity-based information metrics such as image entropy, a measure that is frequently used to determine the information gain in the field of information theory. Specifically, image entropy $E_{I}$ of a given image $I$ is defined as $E_{I}=-\sum_{i} h_{I}(i) \log \left(h_{I}(i)\right)$, where $h_{I}$ denotes the histogram entry of intensity value $i$ in image $I$.

Another intuitive way to determine imaging length is to compare the normalized intensity histogram changes. We hypothesize that if at time $t$ the information has saturated, the information at time $t+1$ should be virtually the same, that is, the normalized intensity distribution remains the same from time $t$ to $t+1$.

Our experiments show that the intensity-based methods generally fail to determine adequate imaging length, as they typically do not take structural information into account. Similarly to FRC, these methods are highly sensitive to noise (Fig. 2I-L). Other information-theoretic methods based on intensities, such as mutual information and $\mathrm{K}-\mathrm{L}$ divergence, share the same problem and fail as well (data not shown).

Structure-based analysis From our preliminary data, we observed that the object contour information tends to stabilize after certain imaging time. We hypothesize that by properly measuring the statistics of the contour information, we should be able to determine adequate imaging length.

We first tested the histogram of oriented gradients (HOG) features method [5], which is widely used to characterize local object appearance and shape within an image. The HOG features are computed by taking the orientation histograms of the image gradients over dense grids of the image. Unlike natural images, however, STORM images are mostly punctate in appearance in the foreground area (Fig. 1A, B), and thus, taking the gradient often results in a number of local random gradient orientations instead of the structural information we aim to extract.

Inspired by the HOG features, we extend the idea of taking the orientation histogram over gradient image to taking the orientation histogram over the orientation map detected by the Gabor filters [6],

$G_{\lambda, \theta, \psi, \sigma, \gamma}\left(x^{\prime}, y^{\prime}\right)=\exp \left(\frac{x^{\prime 2}+\gamma^{2} y^{\prime 2}}{\sigma^{2}}\right) \exp \left(i\left(2 \pi \frac{x^{\prime}}{\lambda}+\psi\right)\right)$,

where $x^{\prime}=x \cos \theta+y \sin \theta, y^{\prime}=-x \sin \theta+y \cos \theta$. Each filter represents a Gaussian kernel function modulated by a complex plane wave whose wavelength and orientation are defined by $\lambda$ and $\theta$, respectively. The parameter $\gamma$ specifies the spatial aspect ratio of the Gabor function, where $\sigma$ is the standard deviation of the Gaussian kernel; we control it by setting $\sigma=0.56 \lambda$. We use eight different orientations, $\theta=$ $k / 8 \pi$, with $k=0,1, \ldots, 7$, and four different scales, $\lambda=$ $2,4,6,8$, resulting in a filter bank of 32 Gabor filters.

After convolving the image with the Gabor filter bank, the orientation histogram is obtained by placing the maximum 

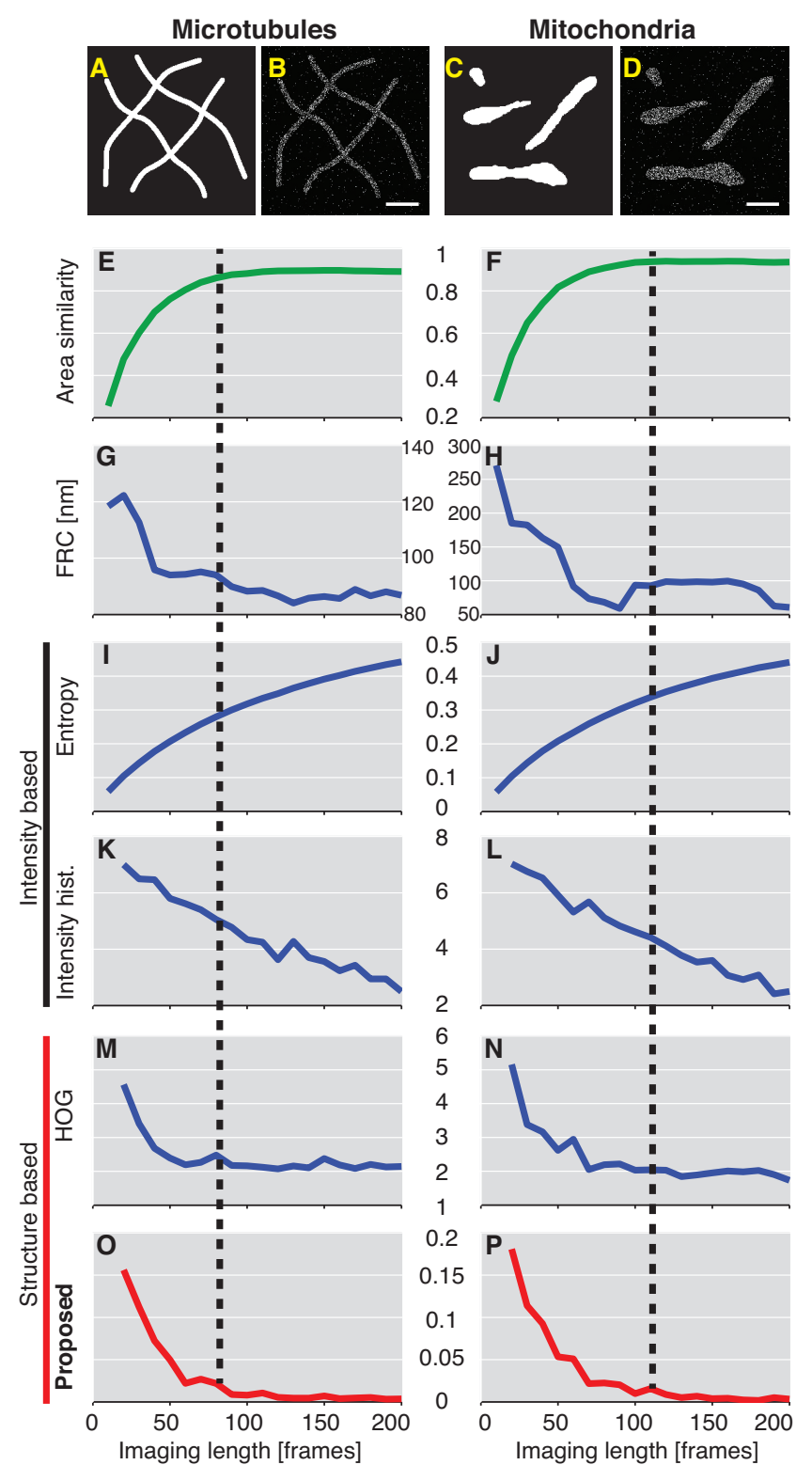

120
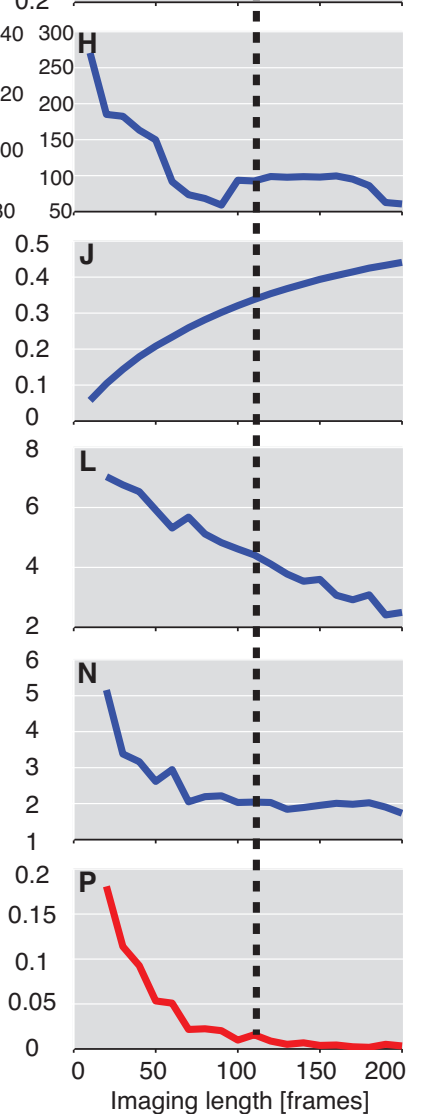

Fig. 2. Comparison of different image-information metrics for determining imaging length. (A, C) Ground truth. (B, D) Simulated STORM images at 200 frames. (E, F) Segmentation results, $(\mathbf{G}, \mathbf{H})$ estimated resolutions by FRC, $(\mathbf{I}, \mathbf{J})$ image entropies, $(\mathbf{K}, \mathbf{L})$ intensity histogram differences, $(\mathbf{M}, \mathbf{N})$ HOG feature differences, and $(\mathbf{O}$, P) proposed orientation histogram differences with different imaging lengths. These methods have also been tested on real STORM data (data not shown). Dashed lines indicate the determined imaging lengths by the proposed method with KS test at p-value $<0.01$. Scale bars, $500 \mathrm{~nm}$.

orientation at each pixel to the orientation bin (Fig. 3). Specifically, let $M_{\theta}(x, y)$ be the filter response at orientation $\theta$, then the maximum orientation map $\theta_{\max }(x, y)$ is

$$
\theta_{\max }(x, y)=\arg \max _{\theta}\left(M_{\theta}(x, y)\right) .
$$

The orientation histogram is then obtained by voting over the

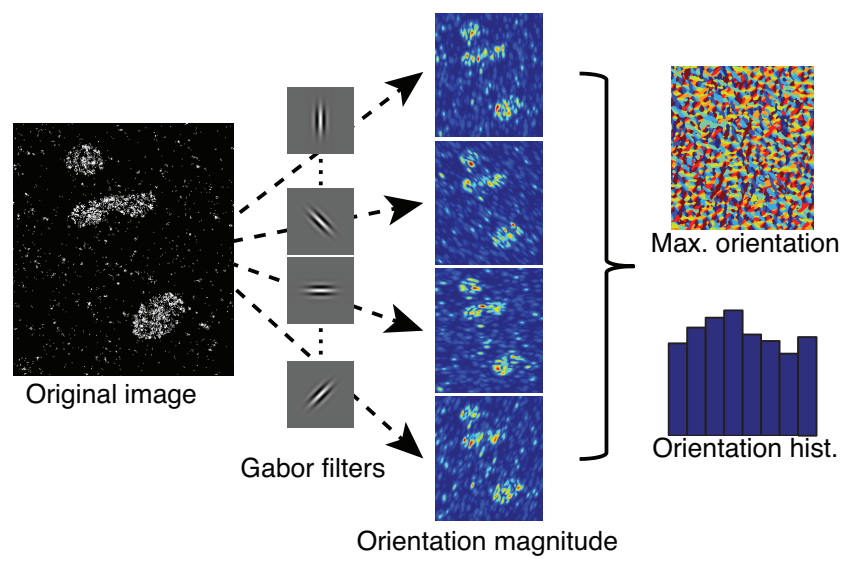

Fig. 3. Gabor filter-based orientation histogram.

entire map $\theta_{\max }(x, y)$ for each orientation as

$$
h_{\theta}=\sum_{x, y} 1_{\theta}\left(\theta_{\max }(x, y)\right),
$$

where $\theta=k / 8 \pi, k=0,1, \ldots, 7$, and $1_{\theta}(\tilde{\theta})$ is the indicator function that is 1 when $\theta=\theta$ and 0 otherwise.

Stopping criterion At each time $t$, we use a statistical test to determine whether there is additional gain in terms of image information. For both the intensity histogram and the proposed orientation histogram, given the histogram $H_{t}$ at time $t$ and $H_{t-1}$ at time $t-1$, we use the Kolmogorov-Smirnov (KS) test to test whether $H_{t}$ and $H_{t-1}$ are from the same distribution; if so, the imaging stops. This statistical test makes histogram-based methods more suitable for setting a global criterion than setting an arbitrary measure, for example, the distance of HOG features.

\section{EXPERIMENTS AND RESULTS}

We performed experiments on both simulated and real STORM images of microtubules and mitochondria. With the ground truth provided from simulated images, we evaluate the performance of determining imaging length by examining the segmentation results.

Simulated data We simulated the molecule detections that are generated by STORM imaging from given ground-truth samples. Two different synthetic sample structures, microtubules and mitochondria (Fig. 2A, C), were produced to simulate common scenarios of biological structures. We assume a uniform fluorophore distribution within the structure, from which the detections of the randomly activated fluorophores are drawn with a photon count sampled from a geometric distribution. For each structure, we simulated the STORM imaging of 200 frames, where in each frame 50 detections inside the structure area and random noise in the background area 
are generated (Fig. 2B, D). This simulated imaging was divided into 20 steps, at which a high-resolution image is reconstructed by accumulating the detections starting with the first frame.

At each step, we compare the results with FRC, the intensity-based methods and the proposed structure-based methods as shown in Fig. 2G-P. To examine the performance of determining imaging length, we used the segmentation of the underlying structure as the standard; in other words, the reconstructed image at an adequate stopping point should lead to good segmentation. Specifically, we segment each reconstructed image using active-mask segmentation algorithm and compare the segmentation result to the ground truth using area similarity [7]. In Fig. 2E, F, the performance of segmentation increases as more frames are used and saturates when the image is reconstructed with more than 90 frames.

As shown in Fig. 2G, H, FRC gives unstable resolution estimates for mitochondria data while the estimate for microtubule data stabilizes only after 130 frames. For intensitybased methods applied to both sample structures, the entropy values increase monotonically, the intensity histogram differences between steps (measured by the $L_{2}$ distance) decrease monotonically, and the KS tests of the intensity histograms give no stopping points (Fig. 2K-L).

For structure-based methods, the results of the proposed method and the HOG feature difference between steps both stabilize after about 100 frames (Fig. 2M-P). Furthermore, the statistical testing for the proposed method (dashed lines in Fig. 2) suggests to stop at about 90 and 110 frames for microtubules and mitochondria respectively, where the segmentation of the corresponding reconstructed image surpasses the accuracy of $90 \%$ compared to the ground truth. Moreover, the suggested imaging length also matches well with the imaging length where segmentation performance saturates, indicating that our method can capture the structural information and determine whether the detections are adequate for imageanalysis methods to be applied.

Real data Next, we tested the proposed method on the real STORM images in Fig. 1. For this data, HeLa cells with fluorescently labeled microtubules and mitochondria were imaged on a Nikon N-STORM system. Our current implementation is written in MATLAB and takes around 1 second for each reconstructed image. In Fig. 4, the proposed method again provides good stopping points at 5,200 frames for microtubules and 30,000 frames for mitochondria, which are both consistent with the visual determination from Fig. 1.

\section{CONCLUSIONS}

We presented a structure-based approach to determine imaging length in super-resolution localization microscopy imaging. The method uses the orientation histogram with a Gabor filter bank to determine the required imaging length based on

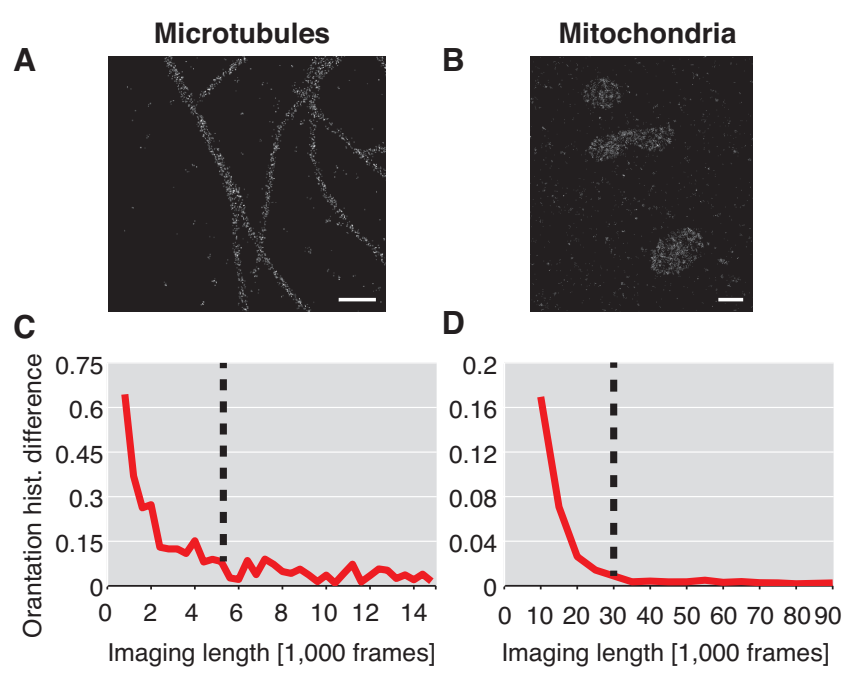

Fig. 4. Results of the proposed method on the real STORM data. Dashed lines indicate the determined imaging lengths by the proposed method with KS test at $\mathrm{p}$-value $<0.01$. Scale bars, $500 \mathrm{~nm}$.

a rigorous statistical test of structural information present in the image. Compared to methods based entirely on image resolution, our method provides a reliable way of determining imaging length. Because of the incorporation of image structural information, this method is particularly well suited to applications that involve subsequent computational image analysis. The proposed approach does not depend on specific image structure and can thus be used in a broad range of biological applications of super-resolution imaging.

\section{REFERENCES}

[1] M.J. Rust, M. Bates, and X. Zhuang, "Sub-diffractionlimit imaging by stochastic optical reconstruction microscopy (STORM)," Nature Methods, vol. 3, no. 10, pp. 793-5, 2006.

[2] E. Betzig, G. H. Patterson, R. Sougrat, O. W. Lindwasser, and et al., "Imaging intracellular fluorescent proteins at nanometer resolution.," Science, vol. 313, no. 5793, pp. 1642-5, 2006.

[3] N. Banterle, K. H. Bui, E. Lemke, and M. Beck, "Fourier ring correlation as a resolution criterion for super-resolution microscopy,” J. Struct. Biol., vol. 183, pp. 363-7, 2013.

[4] R.P.J. Nieuwenhuizen, K.A. Lidke, M. Bates, D.L. Puig, and et al., "Measuring image resolution in optical nanoscopy," Nature Methods, vol. 10, no. 6, pp. 557-62, 2013.

[5] N. Dalal and B. Triggs, "Histograms of oriented gradients for human detection," in Proc. IEEE Int. Conf. Comput. Vis. Pattern Recogn., 2005, vol. 1, pp. 886-893.

[6] J. G. Daugman, "Complete discrete 2-D Gabor transforms by neural networks for image analysis and compression," IEEE Trans. Acoust., Speech, Signal Process., vol. 36, no. 7, pp. 11691179, 1988.

[7] G. Srinivasa, M. C. Fickus, Y. Guo, A. D. Linstedt, and J. Kovačević, "Active mask segmentation of fluorescence microscope images," IEEE Trans. Image Process., vol. 18, no. 8, pp. 1817-1829, 2009. 